\title{
MiRNA Expression Profile of the Myocardial Tissue of Pigs with Coronary Microembolization
}

\author{
Qiang Su ${ }^{a, c}$ Lang Lia Jinmin Zhao ${ }^{b, c}$ Yuhan Sun ${ }^{a}$ Huafeng Yang ${ }^{a}$ \\ aDepartment of Cardiology, The First Affiliated Hospital of Guangxi Medical University, Nanning, \\ bDepartment of Trauma Orthopedic and Hand Surgery, The First Affiliated Hospital of Guangxi Medical \\ University, Nanning, 'Guangxi Key Laboratory of Regenerative Medicine, Guangxi Medical University, \\ Guangxi, China
}

\section{Key Words}

Coronary microembolization $\cdot$ Myocardial damage $\cdot$ MiRNA $\cdot$ Gene expression profile

\begin{abstract}
Background/Aims: Coronary microembolization (CME) is a serious complication of coronary heart disease and is considered as a strong predictor of poor long-term prognosis and major cardiac adverse events. Here, we identified differentially expressed microRNAs (miRNAs) in the myocardial tissue of CME pigs, and predicted and analyzed the possible functions of their target genes. Methods: Twelve Bama mini-pigs were randomly assigned to the sham and CME group ( $n=6$ in each group). The two groups were compared with regard to heart function, area of infarction, cardiomyocyte apoptosis, and myocardial expression of TNF- $\alpha$, IL-1 $\beta$ and IL-6. Further, miRNA chip analysis was used to screen for differentially expressed miRNAs, and the results were validated by real-time PCR. Bioinformatics methods were used to predict and analyze the functions of the target genes of the identified miRNAs. Results: The model CME pigs showed significantly increased expression of TNF- $\alpha$, IL-1 $\beta$ and IL- 6 , as well as microinfarction lesions and cell apoptosis in the myocardial tissue. Thus, the model was established successfully. In the myocardial tissue of the CME pigs, the expression of ssc-miR-92b-5p, ssc-miR-491, ssc-miR-874, ssc-miR-425-3p, ssc-miR-376a-5p, ssc-miR-370, ssc-miR-30c-3p, ssc-miR-493-5p and ssc-miR-323 was significantly increased, whereas the expression of sscmiR-136 and ssc-miR-142-3p was significantly decreased. GO and KEGG pathway analysis indicated that the target genes of these miRNAs are mainly associated with cell proliferation, apoptosis, necrosis, inflammation, and fibrosis. Conclusion: The differentially expressed miRNAs identified in the myocardial tissue of CME pigs could be new biomarkers or potential treatment targets for CME.




\section{Introduction}

Coronary microembolization (CME) refers to coronary microcirculation embolism and myocardial micro-infarction caused by microembolization during spontaneous atherosclerotic plaque rupture or percutaneous coronary intervention (PCI) [1]. The incidence of CME is $15 \%-20 \%$ in the general population, and it can be as high as $30 \%-$ $45 \%$ among high-risk patients [2]. CME can cause immediate "no reflow" or "slow flow" during PCI; thus, it is a strong predictor of poor long-term prognosis and major cardiac adverse events. Moreover, since CME often leads to microcirculation dysfunction, including myocardial micro-infarction, myocardial perfusion-contraction dysfunction, cardiomyocyte apoptosis and necrosis, and coronary reserve decrease, it is considered as an important cause of cardiac dysfunction and arrhythmia $[3,4]$. Studies have confirmed the involvement of several key genes and signal pathways in the pathological process of CME myocardial injury, but the pathogenesis is not yet fully understood [5-7].

Recently, the role and underlying molecular mechanism of microRNAs (miRNAs) in cardiovascular diseases have attracted widespread attention. In particular, the use of miRNAs as a new treatment target has become a hot topic of studies on cardiovascular diseases. miRNAs are endogenous non-coding small RNA molecules made up of 21-25 nucleotides that are widely present in animals, plants, virus, and single-cell organisms. They inhibit mRNA translation or promote mRNA degradation by complete or partial complementary binding to the 3 '-UTR of target mRNAs. miRNAs are essential for cell growth, differentiation, apoptosis, and other biological processes [8-10]. In particular, they regulate the development, differentiation and function of inflammatory cells, and thereby play important roles in cardiovascular immunoinflammatory diseases [11]. Dicer-knockout mice exhibited inhibition of neovascularization, angiogenesis and heart development; further, the expression of heart-specific miRNAs was decreased, and all the mice died of dilated cardiomyopathy and heart failure within $4 \mathrm{~d}$ after birth [12]. Ren et al. [13] found that miR320 participated in myocardial ischemia-reperfusion injury by regulating the expression of heat shock protein 20. Overexpression of miR-320 accelerated necrosis of cardiomyocytes and induced an increase in the size of myocardial infarction lesions, whereas administration of an miR-320 antagonist resulted in a decrease in the size of the lesion [13]. In another miRNA study, Cheng et al. [14]. analyzed the miRNA expression profile in rats with ischemic preconditioning. The results showed that expression of 21 miRNAs was increased and that of 19 miRNAs was decreased. Among the differentially expressed miRNAs, miR-21 showed the most significant increase in expression, and it played a protective role in hypoxia/ reoxygenation-induced apoptosis by regulating the expression of programmed cell death factor 4 [14]. Our previous study also found that overexpression of miR-21 inhibited CMEinduced myocardial inflammatory response and improved heart function via inhibition of the PDCD4/NF- $\kappa B / T N F-\alpha$ signaling pathway [15]. Still, the expression and mechanism of action of other miRNAs involved in CME-induced myocardial injury need to be elucidated.

This study analyzed the miRNA expression profile of the myocardium in pigs with CME by using molecular biotechnologies, and identified the miRNAs involved in CME-induced myocardial injury and their possible targets and functions.

\section{Materials and Methods}

\section{Establishment and evaluation of the pig model of CME}

A total of 12 Bama mini-pigs (male and female, weighing 25-30 kg) were provided by the Department of Animal Science and Technology, Guangxi University. The pigs were randomly assigned to the CME or sham group, with 6 pigs in each group. Animals in the CME group were first anesthetized by intramuscular injection of ketamine hydrochloride (5-10 mg/kg). After 3-5 min, the pigs could not stand steadily and would fall down to the ground. Then, the pigs were rinsed, sterilized, and prepared for surgery. A $51 / 2$ scalp needle was used to puncture and make an indwelling in the ear vein for the maintenance of anesthesia by 
diazepam administered at a dose of $0.5 \mathrm{mg} \cdot \mathrm{kg}^{-1} \cdot \mathrm{h}^{-1}$. The femoral artery on one side was separated, punctured, and implanted with a 6-F arterial sheath. Heparinization was achieved by injecting $200 \mathrm{U} / \mathrm{kg}$ of heparin into the sheath, and it was maintained at a dose of $100 \mathrm{U} \cdot \mathrm{kg}^{-1} \cdot \mathrm{h}^{-1}$. After coronary angiography was performed, the microcatheter was delivered, under the direction of a guiding catheter, to the distal end of the first diagonal branch from the left anterior descending branch. The CME pig model was established by injecting 100, 000 microembolization balls (42- $\mu$ m diameter, Biosphere Medical Inc., USA) (suspended in $1.5 \mathrm{ml}$ of saline) via the microcatheter $[16,17]$, while the sham group was injected with $1.5 \mathrm{ml}$ of saline.

Evaluation of heart function (Philips Sonos 750, USA): In our previous study, we found that heart function was the worst in the pigs at $9 \mathrm{~h}$ after CME induction, so we selected the postoperative $9 \mathrm{~h}$ as the observation period and assessed left ventricle ejection fraction (LVEF), left ventricle end-diastolic diameter (LVEDd), left ventricle shortening fraction (FS), and cardiac output (CO) of pigs in the two groups. The probe frequency was set at $12 \mathrm{MHz}$, and the mean values of data obtained from three cardiac cycles were used for statistical analysis [18]. All echocardiographic examinations were performed by an experienced physician.

Detection of serum cTnI: The BayerACS180 automatic chemiluminescence immunoassay system and the cTnI-specific double-antibody sandwich ELISA kit were used to detect serum cTnI in Bama mini-pigs. The specific steps are as follows: $100 \mu \mathrm{l}$ of acridine ester-labeled anti-cTnI antibody was added to $100 \mu \mathrm{l}$ of serum, and the solution was incubated at $37^{\circ} \mathrm{C}$ for $2.5 \mathrm{~min}$. Then, $200 \mu \mathrm{l}$ of total cTnI solid-phase reagent (anti-cTnIMcAbs covalently bound to magnetic particles) was added and incubated for 5 min. Following this, the reaction phase was separated by washing with ion water, and $300 \mu \mathrm{l}$ each of reagent 1 and reagent 2 (chemiluminescent oxidants) was added for the photochemical reaction and detection of the results.

The animals were sacrificed under anesthesia induced by the injection of $10 \%$ potassium chloride into the ear vein. The chest was opened, the heart was isolated, a small amount of scattered pale myocardium was visible in the left ventricular anterior wall, and the ventricles were sliced in parallel to the atrioventricular groove into six sections, each with a thickness of 5-8 mm. The pathological sections were prepared by fixing $300 \mathrm{mg}$ of left ventricular anterior myocardium in 4\% of neutral formaldehyde, and HE and HBFP staining was performed later to determine the lesion size of the myocardial infarction. HBFP staining is an important method for early diagnosis of myocardial ischemia: ischemic cardiomyocytes and red blood cells are stained red, whereas in normal cardiomyocytes, the cytoplasm is yellow and the nucleus is stained blue. The DMR+Q550 imaging system was used for pathological analysis. For each HBFP-stained section, five fields $(\times 100)$ were randomly selected, and the area of infarction was measured using the planar area method provided by the Leica Qwin program. The area of infarction was presented as the percentage of the lesion area of the total analyzed area, and the mean value was used for statistical analysis [19].

TUNEL analysis of cardiomyocyte apoptosis: All procedures were performed in strict accordance with the instructions provided by the manufacturer. Under a light microscope, the nuclei of apoptotic cells appear brown in color (TUNEL positive). For each slice, 40 non-overlapping fields $(\times 400)$ were selected in the micro-infarction area, the periphery of the micro-infarction area, and the infarction-free area. The number of apoptotic cardiomyocytes as well as total cardiomyocytes was counted, and the apoptotic rate was calculated as the number of apoptotic cardiomyocytes/the number of total cardiomyocytes $\times 100 \%$ [20].

Expression of cleaved caspase-3 proteins in the myocardium by Western blot: Total proteins obtained from cardiac tissues and cardiomyocytes were separated by 10\%-15\% SDS-PAGE and electrotransferred to PVDF membranes (Millipore, Atlanta, GA, US). The membranes were blocked with $5 \%$ bovine serum albumin or non-fat milk for $1.5 \mathrm{~h}$ at room temperature and then incubated at $4^{\circ} \mathrm{C}$ overnight with primary antibodies against cleaved caspase-3. Antibody was obtained from Cell Signaling Technology (Beverly, MA, USA). After washing with TBS containing $0.1 \%$ Tween 20 (TBST) for 5 times, the membranes were incubated with the secondary antibodies conjugated with horseradish peroxidase in TBST for $2 \mathrm{~h}$ at room temperature. The signals were detected with an enhanced chemiluminescence detection system (Pierce, Rockford, IL, USA). The bands for protein amounts were assessed and quantified by using Image Lab software from Bio-Rad.

\section{Total RNA extraction from the myocardial tissue}

The myocardial tissue was treated with $1 \mathrm{ml}$ of Trizol (Invitrogen, USA), ground on ice with a homogenizer, and placed in a $4^{\circ} \mathrm{C}$ freezer for $15 \mathrm{~min}$. Then, $200 \mathrm{ml}$ of chloroform was added to the homogenate, and the mixture was turned upside down several times, placed at room temperature for 2-3 min, and centrifuged at $4^{\circ} \mathrm{C}$ and $12000 \mathrm{~g}$ for $15 \mathrm{~min}$. The suspension was aspirated into a new Eppendorf 


\section{Cellular Physiology Cell Physiol Biochem 2017;43:1012-1024 \begin{tabular}{ll|l} 
and Biochemistry & $\begin{array}{l}\text { DOI: 10.1159/000481699 } \\
\text { Published online: October 02, } 2017\end{array}$ & $\begin{array}{l}\text { (c) } 2017 \text { The Author(s). Published by S. Karger AG, Basel } \\
\text { www.karger.com/cpb }\end{array}$
\end{tabular}}

Su et al.: MiRNA Expression in Coronary Microembolization

tube, to which $500 \mu \mathrm{l}$ of isopropyl alcohol was added, and placed in a $-20^{\circ} \mathrm{C}$ freezer for 15 min. Following this, RNA was precipitated by centrifugation at $4^{\circ} \mathrm{C}$ and $12000 \mathrm{~g}$ for $10 \mathrm{~min}$; the suspension was discarded, and the RNA was washed with 75\% ethanol (prepared with DEPC water). Then, the tube was centrifuged at $4^{\circ} \mathrm{C}$ and $7500 \mathrm{~g}$ for $5 \mathrm{~min}$, the suspension was discarded, and the RNA was washed again. Finally, the RNA was dried at room temperature for 10-20 min and dissolved in DEPC water, and the concentration was measured using a Nanodrop spectrophotometer (ND-1000; Nanodrop Technologies, USA). Agar gel electrophoresis $(1 \%)$ showed that there was no RNA degradation, and the extracted RNA was reversetranscribed for detecting the expression of TNF- $\alpha$, IL-1 $\beta$, IL-6 and miRNAs.

\section{miRNA microarray}

The samples were labeled using the miRCURY ${ }^{\mathrm{TM}} \mathrm{Hy}^{\mathrm{TM}} / \mathrm{Hy} 5^{\mathrm{TM}}$ power labeling kit and hybridized on the miRCURY ${ }^{\mathrm{T}}$ LNA Array (v.18.0). Following the washing steps, the slides were scanned using the Axon GenePix 4000B microarray scanner (Axon Instruments, Foster City, CA). Scanned images were then imported into the GenePix Pro 6.0 software (Axon) for grid alignment and data extraction.

\section{Validation by quantitative real-time PCR}

TNF- $\alpha$, IL-1 $\beta$, IL-6 and the microarray analysis results for specific miRNAs were validated by quantitative real-time PCR (qRT-PCR). Reverse transcription was performed using the SuperScript@III First Strand Synthesis Kit (Life Technologies, Carlsbad, CA). Forward and reverse primers for specific miRNAs were synthesized by KangChen Bio-tech (Shanghai, China). Real-time PCR assays were performed with the SYBR ${ }^{\circledR}$ Select Master Mix (Life Technologies, Carlsbad, CA) on a 7900HT FAST Real-Time PCR System (Life Technologies, Carlsbad, CA). All reactions were run in triplicate and porcine U6 snRNA was used as an endogenous reference. To calculate the expression level differences of miRNAs between samples examined, the relative expression levels were determined using the $2^{-\Delta \Delta C T}$ method.

\section{miRNA targets and pathway analysis}

miRecords, Targetscan, MicroCosm and Miranda were used for target prediction. The Gene Ontology (GO) database and the Kyoto Encyclopedia Genes \& Genomes (KEGG) pathway database were used for functional analysis of the selected miRNAs.

\section{Statistical analysis}

Data were analyzed with SPSS 23.0 (IBM, Chicago, USA). Continuous variables are presented as the mean \pm standard deviation (SD) values. Between-group comparison was performed by Student's t-test. A value of $\mathrm{P}<0.05$ was considered to indicate statistical significance. All experiments conformed to the ethics code of the Chinese Academy of Medical Sciences.

\section{Results}

\section{General characteristics of the sham and CME pigs}

As shown in Table 1, the CME group exhibited a decrease in heart function compared to the sham group: CME pigs had myocardial systolic dysfunction and left ventricular dilation (which manifested as decrease in LVEF, FS and CO, $P<0.05)$, and had increased LVEDd $(P<$ 0.05). Moreover, when compared with the sham pigs, the CME pigs had significantly higher serum cTnI levels $(0.196 \pm 0.015 \mathrm{ng} / \mathrm{ml}$ vs. $0.037 \pm 0.004 \mathrm{ng} / \mathrm{ml}, P<0.05)$. HE staining indicated that in the $\mathrm{CME}$ group, cells in the microinfarction area had a dissolved nucleus or the nucleus had disappeared altogether. Further, the cytoplasm was colored red, and the peripheral myo-

Table 1. Changes in cardiac function $(\bar{x} \pm s)$, LVEF: left ventricle ejection fraction; CO: cardiac output; LVEDd: left ventricular end-diastolic diameter; LVFS: left ventricle fractional shortening. ${ }^{*} \mathrm{P}<0.05$ vs. the sham group

\begin{tabular}{lccccc}
\hline Group & $\mathrm{n}$ & LVEF (\%) & LVFS (\%) & CO (L/min) & LVEDd (mm) \\
\hline Sham & 6 & $68.33 \pm 2.61$ & $41.86 \pm 5.83$ & $3.79 \pm 0.77$ & $3.31 \pm 0.38$ \\
CME & 6 & $50.87 \pm 3.53^{*}$ & $27.63 \pm 7.89^{*}$ & $2.81 \pm 0.82^{*}$ & $4.94 \pm 0.56^{*}$ \\
\hline
\end{tabular}




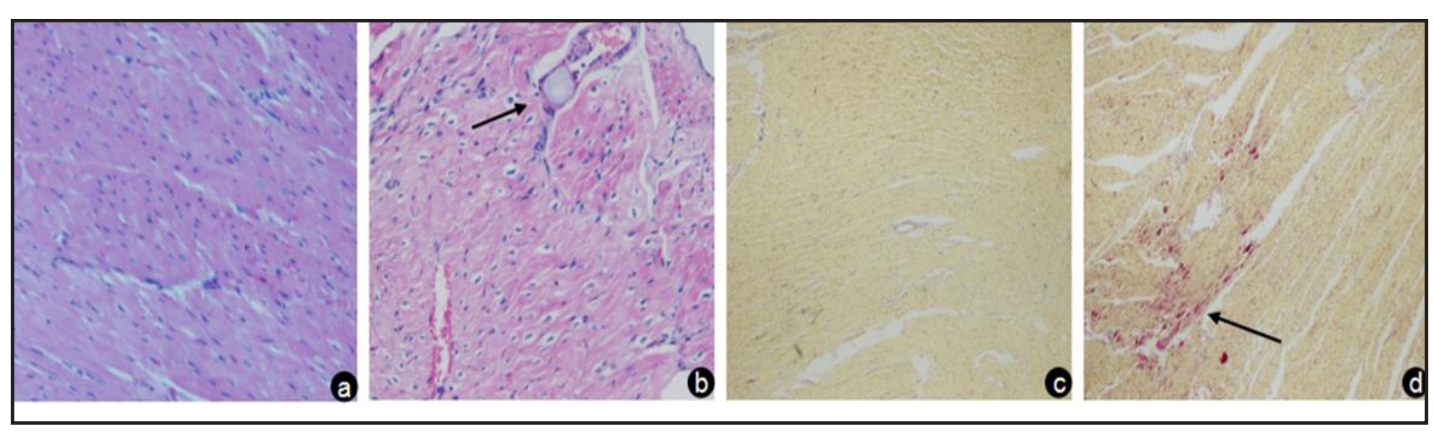

Fig. 1. HE and HBFP staining. (a): HE staining of sham group. (b): HE staining of micro-infarction lesions in the local myocardium of CME pigs. The arrow indicates a microembolization ball ( $\times 400)$. (c) and (d): HBFP staining in the sham and CME group. The ischemic myocardium is stained red, and the arrow indicates a micro-infarction lesion $(\times 200)$.

cardium showed edema and degeneration, accompanied by inflammatory cell infiltration and erythrocyte exudation. HBFP staining indicated that in the sham group, subendocardial ischemia was occasionally observed, but no obvious infarction was observed. Under a light microscope, microembolization balls could be observed in the micro-arteries of CME pigs. Micro-infarction lesions were formed in the peripheral, and were mostly wedge-shaped and locally distributed (Fig. 1). TUNEL staining indicated that after CME induction, apoptosis of cardiomyocytes mainly occurred in the micro-infarction area and the periphery. With regard to the sham group, cardiomyocyte apoptosis was occasionally observed in the endocardium. The CME pigs showed a significantly higher apoptosis rate $(5.24 \pm 1.08 \%$ vs. $0.36 \pm 0.10 \%$, $P<0.05$, Fig. 2) as well as significantly higher TNF- $\alpha$, IL-1 $\beta$ and IL-6 expression $(P<0.05$, Fig. $3)$ than the sham group pigs. In addition, the expression level of cleaved-caspase-3 protein in the CME group increased significantly compared to the sham group $(P<0.05$, Fig. 4$)$. Thus, the CME pig model was established successfully.

\section{miRNA expression profile in the myocardium}

Differential expression analysis of miRNA showed that 11 miRNAs were differentially expressed at a significant level in the CME model, compared with the sham group $(>2$ - or $<0.5$-fold change in expression, $P<0.05$ ). Of them, 9 miRNAs showed an increase in their expression, namely, ssc-miR92b-5p, ssc-miR-491, ssc-

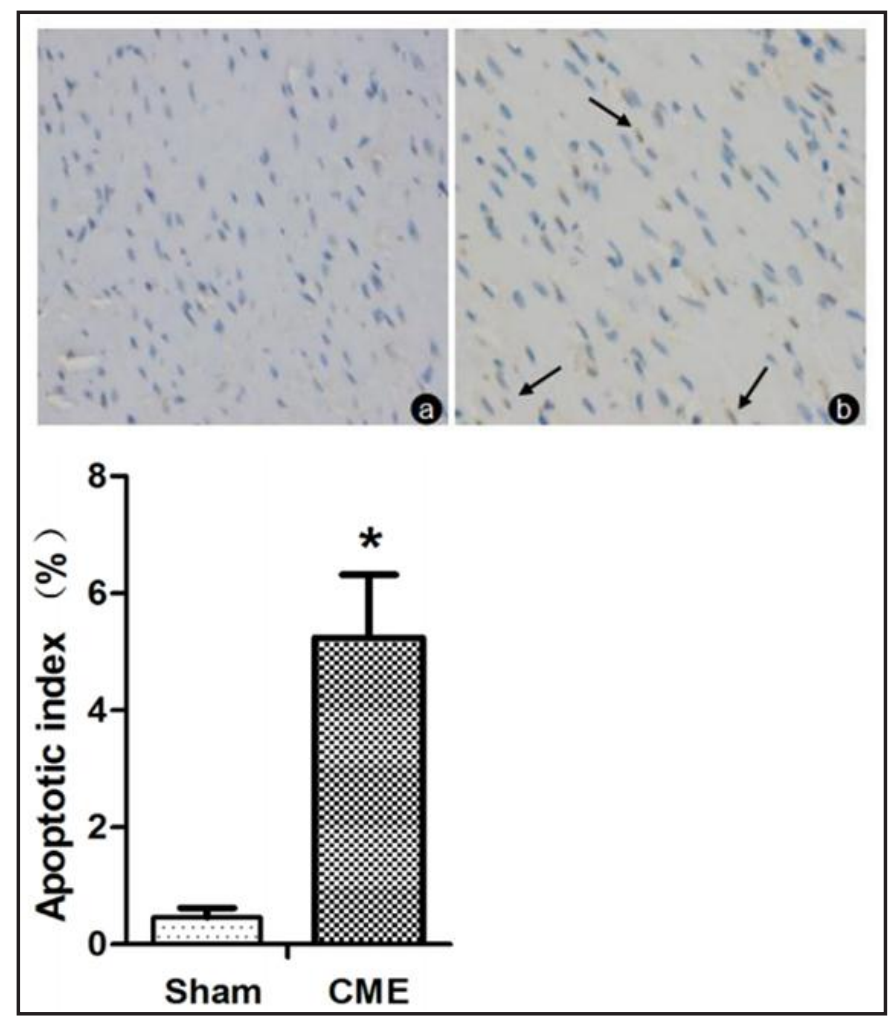

Fig. 2. TUNEL detection of cardiomyocyte apoptosis. (a) and (b) depict staining in the sham and CME group: the nucleus of apoptotic cardiomyocytes is stained brown, while the nucleus of normal cardiomyocytes is stained blue. The arrows indicate the nuclei of apoptotic cardiomyocytes $(\times 400)$. ${ }^{*} \mathrm{P}<0.05$ vs. the sham group.

\section{KARGER}




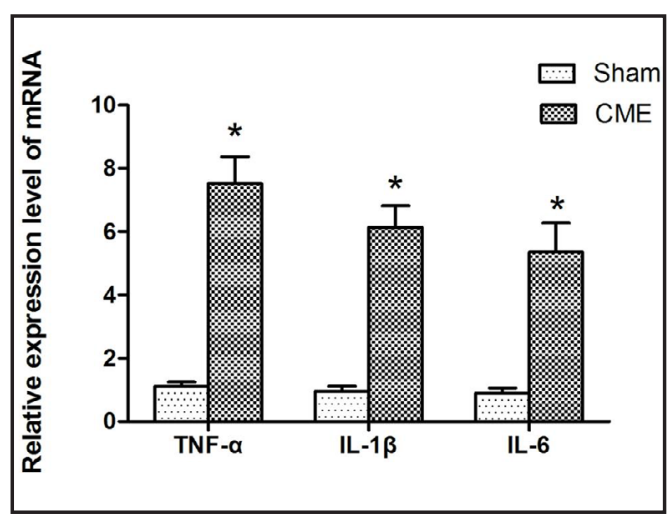

Fig. 3. qRT-PCR detection of TNF- $\alpha$, IL-1 $\beta$ and IL-6 mRNA expression. ${ }^{*} \mathrm{P}<0.05$ vs. the sham group.

Table 2. miRNAs with significantly different expression in the myocardial tissue of CME pigs

\begin{tabular}{lccc}
\hline & miRNA & Fold change & P \\
\hline Upregulation & ssc-miR-92b-5p & 3.760 & 0.011 \\
& ssc-miR-491 & 2.119 & 0.000 \\
& ssc-miR-874 & 3.969 & 0.023 \\
& ssc-miR-425-3p & 2.069 & 0.002 \\
& ssc-miR-376a-5p & 4.688 & 0.029 \\
& ssc-miR-370 & 2.007 & 0.033 \\
& ssc-miR-30c-3p & 3.784 & 0.005 \\
& ssc-miR-493-5p & 6.417 & 0.028 \\
& ssc-miR-323 & 4.754 & 0.045 \\
Downregulation & ssc-miR-136 & 0.491 & 0.043 \\
& ssc-miR-142-3p & 0.444 & 0.007 \\
\hline
\end{tabular}

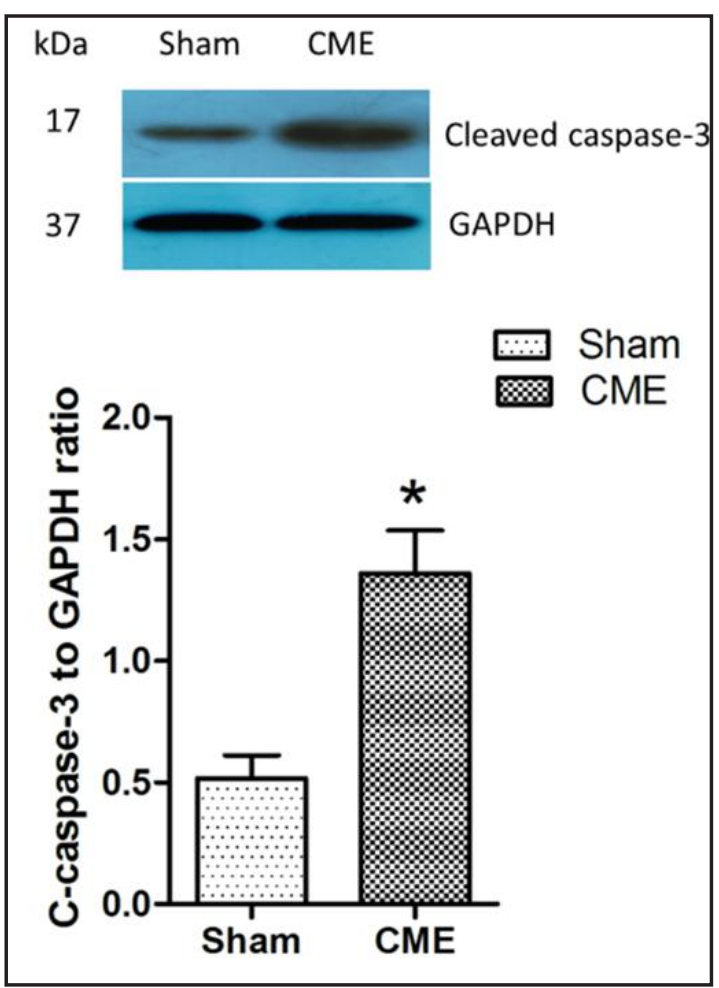

Fig. 4. Western blot detection of cleaved caspase-3 protein expression. ${ }^{*} \mathrm{P}<0.05$ vs. the sham group.

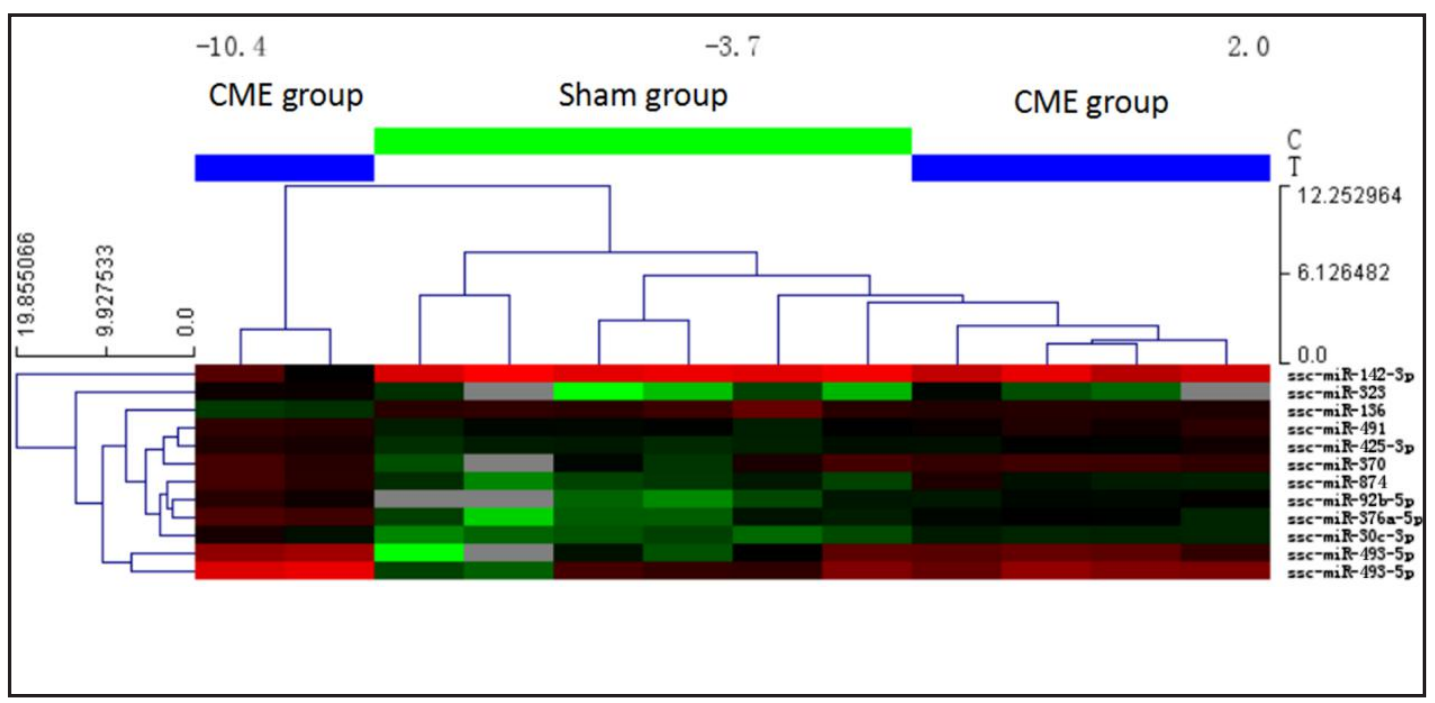

Fig. 5. Hierarchical clustering of the differentially expressed miRNAs. Red indicates relatively high expression, and green indicates relatively low expression.

miR-874, ssc-miR-425-3p, ssc-miR-376a-5p, ssc-miR-370, ssc-miR-30c-3p, ssc-miR-493-5p and ssc-miR-323, and 2 miRNAs showed a decrease in their expression, namely, ssc-miR-136 and ssc-miR-142-3p (Table 2). The volcano plot depicting the differential expression of these miRNAs is shown in Fig. 6, and the hierarchical clustering is shown in Fig. 5. 


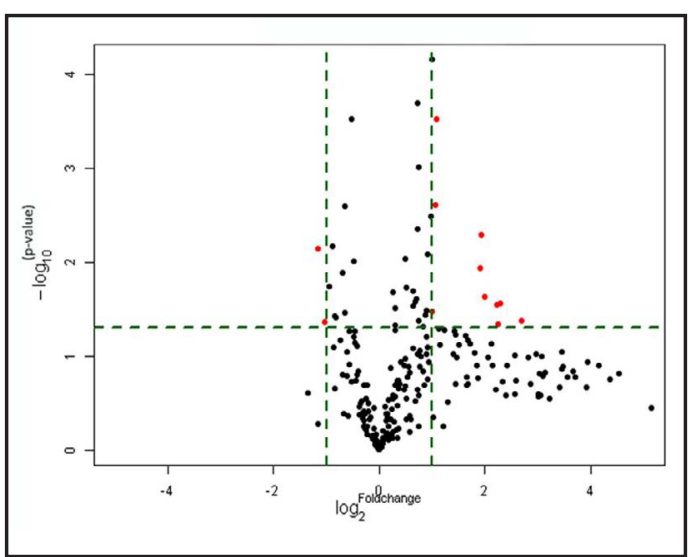

Fig. 6. Volcano plot of the differentially expressed miRNAs. The vertical lines correspond to 2.0fold upregulation and downregulation, and the horizontal line represents a p-value of 0.05 . The red point in the plot represents the differentially expressed miRNAs with statistical significance.

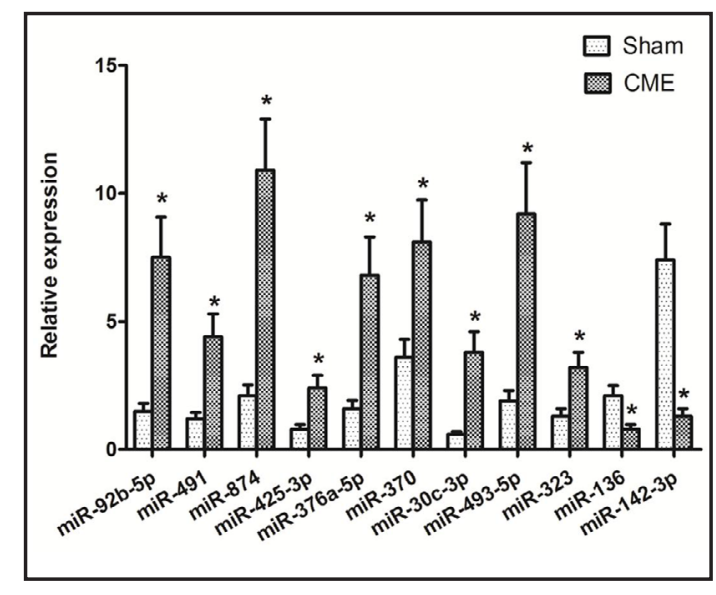

Fig. 7. Verification of the expression of the identified miRNAs with differential expression by qRT-PCR. ${ }^{*} \mathrm{P}<0.05$ vs. the sham group.

Validation of miRNA expression by $q R T-P C R$

Fig. 7 shows the results of qRT-PCR analysis: among the 11 candidate miRNAs, 9 showed an increase in expression: ssc-miR-92b-5p, ssc-miR-491, ssc-miR-874, ssc-miR-425-3p, sscmiR-376a-5p, ssc-miR-370, ssc-miR-30c-3p, ssc-miR-493-5p and ssc-miR-323. Two miRNAs showed a decrease in their expression: ssc-miR-136 and ssc-miR-142-3p. The expression of these miRNAs was significantly different between the CME and sham groups $(P<0.05)$, and the results were consistent with those of miRNA microarray analysis. Thus, these miRNAs may play a role in CME-associated myocardial injury.

GO and KEGG pathway analysis of the 11 differentially expressed miRNAs

To determine the targets of the 11 differentially expressed miRNAs, we performed GO analysis with the following categories: biological process (BP), cellular component (CC) and molecular function (MF). The results showed that in the BP analysis, the targets were mostly involved in cellular processes (2725), which were followed by metabolic processes (2052) and biological regulations (1907) (Fig. 8A). In the CC analysis, the targets were mostly located in the cellular components and cell (2701 for both); this was followed by the intracellular (2400) and intracellular components (2274) (Fig. 8B). The MF analysis showed that the targets mainly participated in binding (2722), protein binding (2133) and catalytic activity (1204) (Fig. 8C). The distribution of these genes indicated differential gene expression during the development of CME (data not shown).

KEGG pathway analysis also focused on the targets of these 11 differentially expressed miRNAs. The results showed that the target genes were involved in a total of 74 signaling pathways, and the signaling targets of these genes were also determined (data not shown). As shown in Fig. 9, leukocyte transendothelial migration, HTLV-I infection, inositol phosphate metabolism, the T-cell receptor signaling pathway, endocytosis, the TGF-beta signaling pathway, the NF-kappa B signaling pathway, the PI3K-Akt signaling pathway, the Toll-like receptor signaling pathway and apoptosis might be the pathways involved in the development of CME. 
A

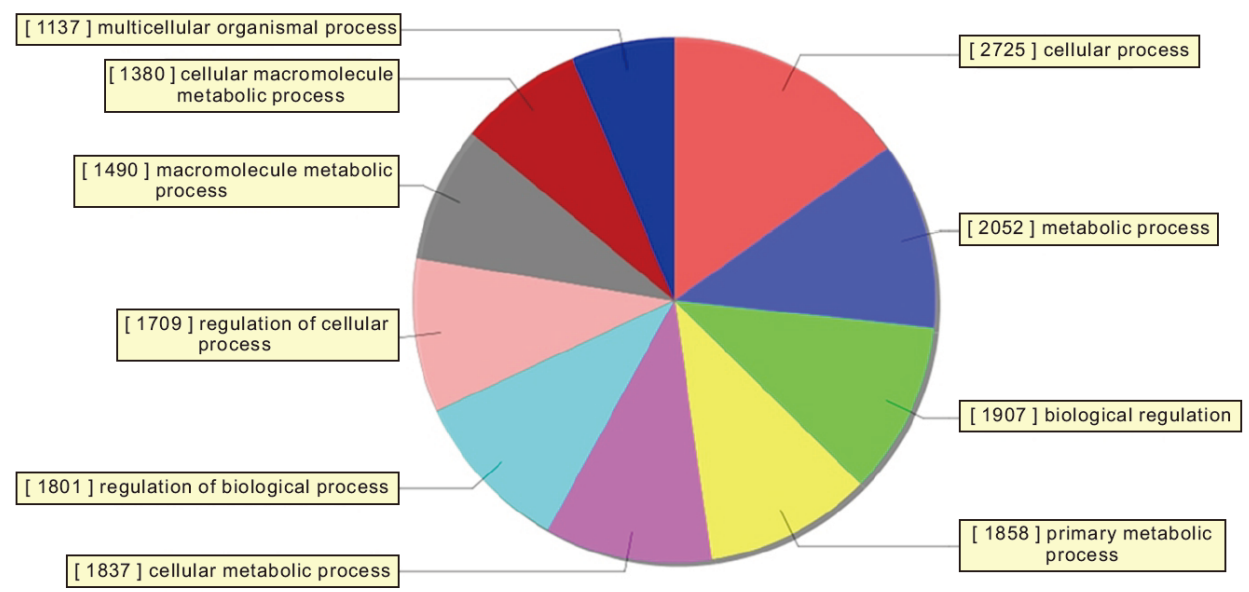

B

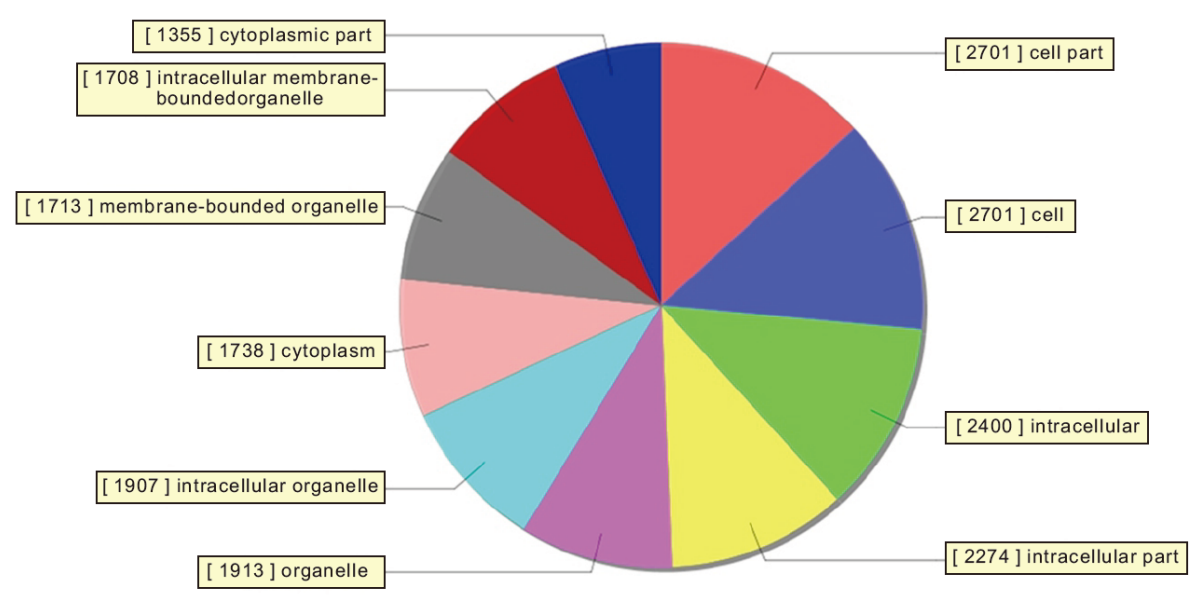

C

[ 487 ] nucleoside phosphate binding

[487] organic cyclic compound binding [487] nucleotide binding

[529] small molecule binding

[633] metal ion binding

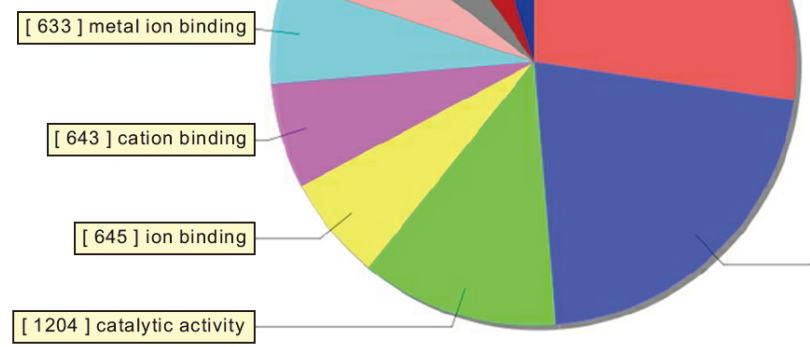

Fig. 8. Distribution of the target genes of differentially expressed miRNAs. (A): biological process, (B): cellular component, (C): molecular function. 


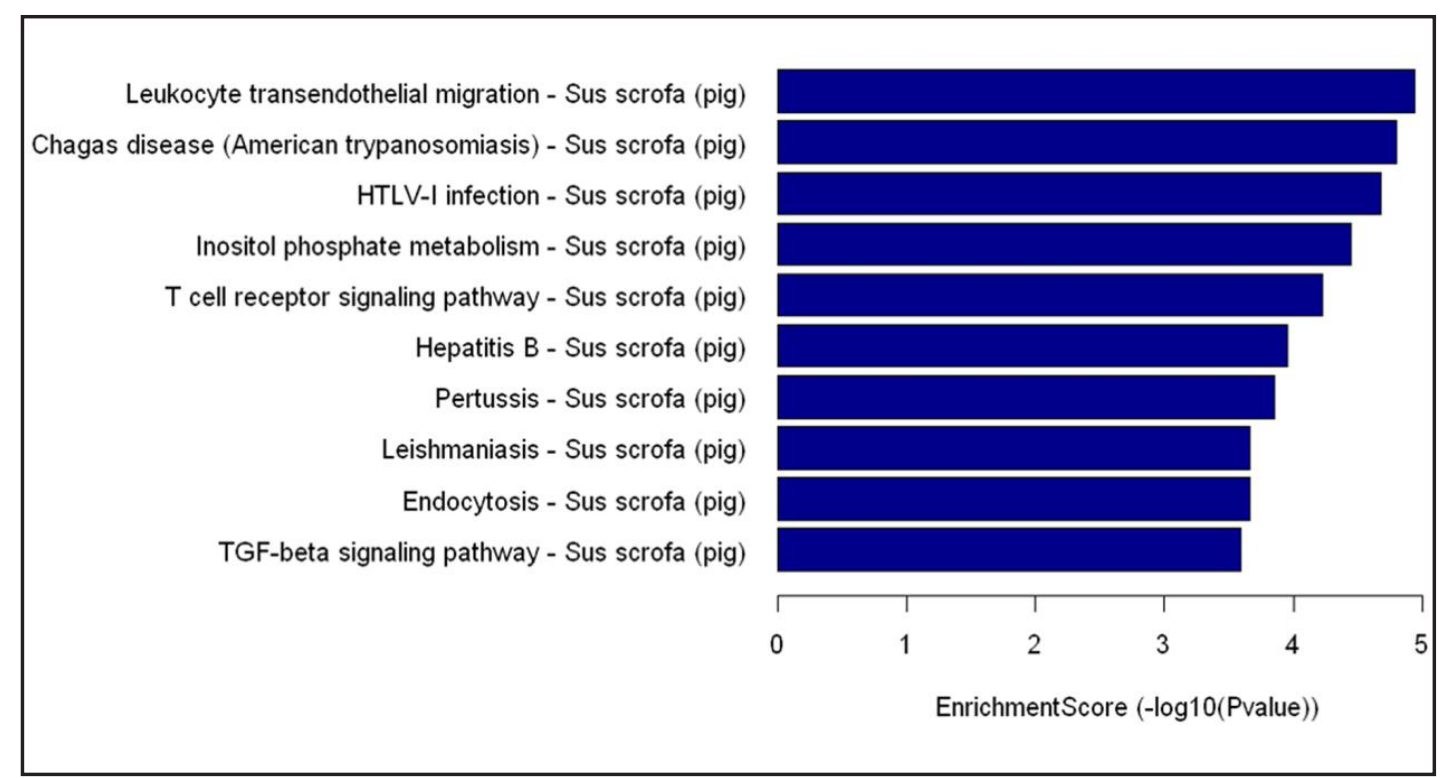

Fig. 9. Pathway analysis of the target genes of the differentially expressed miRNAs. The vertical axis represents the pathway categories, and the horizontal axis represents the negative logarithm of the $p$ value $(-\log p)$, which represents the significance level of the pathways.

\section{Discussion}

In this study, we established a pig CME model by intracoronary injection of microembolization balls using the microcatheter intervention technique. Heart function was evaluated at $9 \mathrm{~h}$ after CME induction, and the results showed that when compared with the sham group, pigs in the CME group had significantly decreased heart function and increased myocardial enzyme levels. Moreover, under a light microscope, microembolization balls could be observed in the arterioles. Further, micro-infarction lesions were formed at the periphery, and clear necrosis of cardiomyocytes was also observed. In addition, the CME group also showed significantly increased expression of inflammatory cytokines (TNF- $\alpha$, IL$1 \beta$ and IL-6) compared to the sham group. The signal chain of TNF- $\alpha$ reactive oxygen species may well causally contribute to the observed contractile dysfunction [21]. These changes were in line with the pathological features of CME, and they properly simulated clinical CME; thus, this model was successfully established.

CME is a serious complication of coronary heart disease that is particularly observed in patients receiving interventional therapy for thrombosis. With regard to the mechanism of CME-induced myocardial injury, previous studies have reported that after CME, coronary blood flow only shows a transient decrease, but the contractility of the local myocardium declines progressively [22]. Further, CME is frequently associated with myocardial inflammatory responses characterized by significant infiltration of white blood cells, monocytes and macrophages at the periphery of micro-infarction lesions [23]. However, CME causes necrosis in cardiomyocytes only to a small extent, and the transient decrease in coronary blood flow only has a relatively minor effect [23]. Glucocorticoid treatment can significantly reduce the level of local inflammatory response and the amount of inflammatory cytokines, as well as cardiac function [24]. These results indicate that CME-induced myocardial damage may not be significantly associated with the decrease in coronary blood flow or necrosis of cardiomyocytes, but it may be associated with the development of local inflammatory responses. Our previous animal studies also showed that in the myocardium of CME models, massive infiltration of inflammatory cells was observed at the periphery of micro-infarction lesions, and that the release of large amounts of inflammatory cytokines caused local myocardial inflammation, which is the key to post-CME myocardial damage and progressive 
cardiac dysfunction $[25,26]$. Besides, another study found that cardiomyocyte necrosis also occurred at the periphery of micro-infarction lesions, and that microembolization-induced cardiomyocyte apoptosis might play a role in myocardial injury [27].

The mechanisms of CME-induced myocardial damage mainly include inflammation and apoptosis. However, since this disease is associated with multiple factors, new regulatory factors that are of significant importance need to be identified. Studies in recent years have found that miRNAs, as a post-transcriptional regulatory factor, can simultaneously target multiple mRNAs involved in different signaling pathways, and thereby regulate gene expression either at the transcriptional or at the post-transcriptional level [28]. Thus, various intracellular cytokines and signaling pathways work in synergy under miRNA regulation [29]. In the heart, miRNAs were also found to regulate various physiological or pathological processes of the myocardium, including cardiac remodeling, cardiac development, myocardial fibrosis, myocarditis, and cardiomyocyte apoptosis [30,31]. Based on these findings, in this study, by analyzing the differential expression of miRNAs in the myocardium of CME pigs, we aimed to identify the miRNAs associated with CME-induced myocardial damage in order to determine the potential mechanisms by which CME causes myocardial damage.

We preliminarily selected differentially expressed miRNAs in the myocardial tissue of CME pigs by using miRNA microarray. The results showed that in the myocardium of CME pigs, a total of 9 miRNAs exhibited significantly increased expression ( $>2$-fold) and 2 miRNAs showed significantly decreased expression $(<0.5$-fold). This indicated that when compared with normal myocardial tissue, CME-damaged myocardial tissue had a unique miRNA expression profile, and that these differentially expressed miRNAs might participate in the development of CME-induced myocardial damage. Following this, qRT-PCR was performed to verify the microarray results, and it was confirmed that the 11 miRNAs did show significantly different expression in CME-damaged myocardial tissues. The 9 miRNAs with increased expression were ssc-miR-92b-5p, ssc-miR-491, ssc-miR-874, ssc-miR-4253p, ssc-miR-376a-5p, ssc-miR-370, ssc-miR-30c-3p, ssc-miR-493-5p and ssc-miR-323, and the 2 miRNAs with decreased expression were ssc-miR-136 and ssc-miR-142-3p.

miR-370, miR-323, miR-136, miR-142-3p, miR-92b-5p, miR-874 and miR-30c-3p play important roles in lipid metabolism, myocardial apoptosis, myocardial fibrosis, and myocardial necrosis. Significantly increased levels of miR-370 and miR-323 are observed in the circulating blood of patients with coronary heart disease; these miRNAs may therefore be associated with the regulation of lipid metabolism and could be used as a reliable and stable predictor of coronary heart disease [32, 33]. Besides, Wang et al. suggested that miR-370 was involved in the proliferation of cardiac microvascular endothelial cells, in which it exerted an important regulatory role [34]. In addition, the study by Ghanbari et al. identified a significant association between miR-136 and cardiovascular metabolism: the SNP rs1059611 was located in the binding site of miR-136, where it showed significant correlation with the level of blood lipids [35]. Wang et al. used $\mathrm{H}_{2} \mathrm{O}_{2}$ to induce myocardial damage, and found that the expression of miR-874 was significantly increased in damaged cells. Knockout of miR-874 alleviated cardiomyocyte necrosis and apoptosis via regulation of the target gene caspase-8. The study indicated that inhibitors of miR-874 could be useful in the treatment of heart failure via decreasing cardiomyocyte necrosis [36]. Recent studies have also reported that miR-30c-3p and miR-142-3p may participate in the development of myocardial fibrosis by regulating the TGF- $\beta 1 / \mathrm{Smad} 3$ signaling pathway $[37,38]$. Thus, of the 11 identified miRNAs, the roles of miR-370, miR-323, miR-136, miR-142-3p, miR-92b-5p, miR-874 and miR-30c-3p in cardiovascular diseases have been clarified in previous studies, but the functions or mechanisms of miR-491, miR-425, miR-493, and miR376a have not been reported. Furthermore, there are no reports on whether these 11 miRNAs are involved in the development and progression of CME-induced myocardial damage. Thus, the present study is the first to link these miRNAs with CME, and the first to link the four novel miRNAs with cardiac function.

miRNA target prediction and function analysis can help reveal the possible functions and mechanisms of abnormal miRNAs in CME-associated myocardial damage. Thus, in 
this study, GO analysis and KEGG pathway analysis were performed on the differentially miRNAs that were identified. The results showed that the target genes of the differentially expressed miRNAs are mainly involved in cellular processes, metabolic processes and biological regulation. Further, most of them are located in the cell part, cell, intracellular and intracellular part, and their functions mainly included binding, protein binding and catalytic activity. These findings were indicative of the genetic network regulated by these miRNAs during CME-induced myocardial damage. Moreover, with regard to CME-associated signaling pathways, the targets of these miRNAs are possibly involved in the T cell receptor signaling pathway, TGF-beta signaling pathway, NF-kappa B signaling pathway, PI3K-Akt signaling pathway, Toll-like receptor signaling pathway, etc. Since these pathways are related to inflammation and apoptosis, we speculate that the identified differentially expressed miRNAs might influence CME-induced myocardial damage by regulating the target genes involved in these pathways.

Limitations of this study: Our study is limited to a small number of pigs and requires prospective confirmation in larger cohorts. Although this study found the differentially expressed miRNAs in myocardial tissue after CME for the first time, the target genes and the specific regulatory mechanism of the corresponding miRNAs had not been studied deeply. The causal association of microRNA expression to a biological function such as cardiomyocyte death or salvage remains largely enigmatic. In addition, this study did not carry out in-depth study the cell type of the myocardial tissue with different expression of microRNAs, which would be our further direction in the future.

In conclusion, this study identified a total of 11 differentially expressed miRNAs in the myocardial tissue of CME pigs by using miRNA microarray. Apart from the miRNAs that have been reported previously, we also found several new miRNAs that are associated with CME. However, the targets of these miRNAs and how they influence the expression of target proteins during CME-induced myocardial damage are still unknown. The mechanism by which these miRNAs affect the structures and functions of the damaged myocardium will be discussed at a molecular level in our future studies, with the help of which we hope to provide new insight into the prevention and treatment of CME-induced myocardial damage.

\section{Acknowledgements}

This study was supported by a grant from National Natural Science Foundation of China (Grant No. 81600283) and Guangxi Natural Science Foundation (Grant No. 2016GXNSFBA380022).

\section{Disclosure Statement}

This paper has not been published elsewhere in whole or in part. All authors have read and approved the content, and agree to submit for consideration for publication in the journal. There are no any ethical/legal conflicts involved in the article.

\section{References}

1 Heusch G, Kleinbongard P, Böse D, Levkau B, Haude M, Schulz R, Erbel R: Coronary microembolization: from bedside to bench and back to bedside. Circulation 2009;120:1822-1836.

2 Böse D, Birgelen CV, Zhou XY, Schmermund A, Philipp S, Sack S, Konorza T, Möhlenkamp S, Leineweber $\mathrm{K}$, Kleinbongard P: Impact of atherosclerotic plaque composition on coronary microembolization during percutaneous coronary interventions. Basic Res Cardiol 2008;103:587-597.

-3 Jaffe R, Charron T, Puley G, Dick A, Strauss BH: Microvascular obstruction and the no-reflow phenomenon after percutaneous coronary intervention. Circulation 2008;117:3152-3156. 


\section{Cellular Physiology Cell Physiol Biochem 2017;43:1012-1024

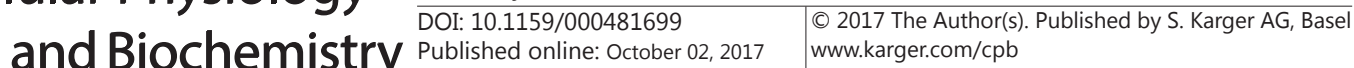

4 Brosh D, Assali AR, Mager A, Porter A, Hasdai D, Teplitsky I, Rechavia E, Fuchs S, Battler A, Kornowski R: Effect of no-reflow during primary percutaneous coronary intervention for acute myocardial infarction on six-month mortality. Am J Cardiol;2007;99:442-445.

5 Liu T, Zhou Y, Liu YC, Wang JY, Su Q, Tang ZL, Li L: Coronary Microembolization Induces Cardiomyocyte Apoptosis Through the LOX-1-Dependent Endoplasmic Reticulum Stress Pathway Involving JNK/P38 MAPK. Can J Cardiol 2015;31:1272-1281.

6 Wang X, Lu Y, Sun Y, He W, Liang J, Li L: TAK-242 Protects Against Apoptosis in Coronary Microembolization-Induced Myocardial Injury in Rats by Suppressing TLR4/NF-кB Signaling Pathway. Cell Physiol Biochem 2017;41:1675-1683. Heusch G: The Coronary Circulation as a Target of Cardioprotection. Circ Res 2016; 118:1643-1658. Li X, Zhi Z, Li Q Xu Q, Xie J, Hao H, Luo G, Liao W, Bin J, Huang X: Inhibition of microRNA-497 ameliorates anoxia/reoxygenation injury in cardiomyocytes by suppressing cell apoptosis and enhancing autophagy. Oncotarget 2015;6:18829-18844.

-9 Raffort J, Lareyre F, Clement M, Mallat Z: Micro-RNAs in abdominal aortic aneurysms: insights from animal models and relevance to human disease. Cardiovasc Res 2016;110:165-177.

10 Zhu J, Kang Y, Qian W, Guo J, Shi H, Ma L, Liu H, Wei G, Zou Y, Ge J: Ischemic Postconditioning-Regulated miR-499 Protects the Rat Heart Against Ischemia/Reperfusion Injury by Inhibiting Apoptosis through PDCD4. Cell Physiol Biochem 2016;39:2364-2380.

11 Condorelli G, Latronico MV, Cavarretta E: microRNAs in cardiovascular diseases: current knowledge and the road ahead. J Am Coll Cardiol 2014;63:2177-2187.

12 Chen JF, Murchison EP, Tang R, Callis TE, Tatsuguchi M, Deng Z, Rojas M, Hammond SM, Schneider MD, Selzman CH: Targeted deletion of Dicer in the heart leads to dilated cardiomyopathy and heart failure. Proc Natl Acad Sci U S A 2008;105:2111-2116.

13 Ren XP, Wu J, Wang X, Sartor MA, Qian J, Jones K, Nicolaou P, Pritchard TJ, Fan GC: MicroRNA-320 is involved in the regulation of cardiac ischemia/reperfusion injury by targeting heat-shock protein 20. Circulation 2009;119:2357-2366.

14 Cheng Y, Zhu P, Yang J, Liu X, Dong S, Wang X, Chun B, Zhuang J, Zhang C: Ischaemic preconditioningregulated miR-21 protects heart against ischaemia/reperfusion injury via anti-apoptosis through its target PDCD4. Cardiovasc Res 2010;87:431-439.

-15 Su Q Li L, Liu Y, Zhou Y, Wang J, Wen W: Ultrasound-targeted microbubble destruction-mediated microRNA-21 transfection regulated PDCD4/NF- $\kappa$ B/TNF- $\alpha$ pathway to prevent coronary microembolization-induced cardiac dysfunction. Gene Ther 2015;22:1000-1006.

16 Su Q Li L, Liu T, Wang J, Zhou Y, Liu Y: Effects of atorvastatin on PDCD4/NF- $\kappa$ B/TNF- $\alpha$ signaling pathway during coronary microembolization of miniature pigs. Exp Mol Pathol 2015;99:564-569.

17 Nassenstein K, Breuckmann F, Bucher C, Kaiser G, Konorza T, Schäfer L, Konietzka I, De GA, Heusch G, Erbel R: How much myocardial damage is necessary to enable detection of focal late gadolinium enhancement at cardiac MR imaging? Radiology 2008;249:829-835.

18 Su Q Li L, Zhou Y, Wang J, Liu Y, Ma G: Induction of myocardial PDCD4 in coronary microembolizationrelated cardiac dysfunction: evidence from a large-animal study. Cell Physiol Biochem 2014;34:533-542.

19 Wang J, Chen H, Zhou Y, Su Q, Liu T, Wang XT, Li L: Atorvastatin Inhibits Myocardial Apoptosis in a Swine Model of Coronary Microembolization by Regulating PTEN/PI3K/Akt Signaling Pathway. Cell Physiol Biochem 2016;38:207-219.

-20 Chen ZW, Qian JY, Ma JY, Chang SF, Yun H, Jin H, Sun AJ, Zou YZ, Ge JB: TNF- $\alpha$-induced cardiomyocyte apoptosis contributes to cardiac dysfunction after coronary microembolization in mini-pigs. J Cell Mol Med 2015;18:1953-1963.

21 Canton M, Skyschally A, Menabò R, Boengler K, Gres P, Schulz R, Haude M, Erbel R, Lisa FD, Heusch G: Oxidative modification of tropomyosin and myocardial dysfunction following coronary microembolization. Eur Heart J 2006;27:875-881.

22 Dörge H, Neumann T, Behrends M, Skyschally A, Schulz R, Kasper C, Erbel R, Heusch G: Perfusioncontraction mismatch with coronary microvascular obstruction: role of inflammation. Am J Physiol Heart Circ Physiol 2000;279:H2587-H2592.

-23 Thielmann M, Dörge H, Martin C, Belosjorow S, Schwanke U, van de Sand A, Konietzka I, Büchert A, Krüger A, Schulz R: Myocardial dysfunction with coronary microembolization. Circ Res 2002;90:807-813. 


\section{Cellular Physiology Cell Physiol Biochem 2017;43:1012-1024 \begin{tabular}{l|l} 
DOI: 10.1159/000481699 & $\begin{array}{l}\text { O 2017 The Author(s). Published by S. Karger AG, Basel } \\
\text { www.karger.com/cpb }\end{array}$
\end{tabular}}

Su et al.: MiRNA Expression in Coronary Microembolization

24 Skyschally A, Haude M, Dörge H, Thielmann M, Duschin A, van de Sand A, Konietzka I, Büchert A, Aker S, Massoudy P: Glucocorticoid treatment prevents progressive myocardial dysfunction resulting from experimental coronary microembolization. Circulation 2004;109:2337-2342.

-25 Lu Y, Li L, Zhao X, Huang W, Wen W: Beta blocker metoprolol protects against contractile dysfunction in rats after coronary microembolization by regulating expression of myocardial inflammatory cytokines. Life Sci 2011;88:1009-1015.

-26 Li L, Zhao X, Lu Y, Huang W, Wen W: Altered expression of pro- and anti-inflammatory cytokines is associated with reduced cardiac function in rats following coronary microembolization. Mol Cell Biochem 2010;342:183-190.

27 Liu T, Zhou Y, Wang JY, Su Q, Tang ZL, Liu YC, Li L: Coronary Microembolization Induces Cardiomyocyte Apoptosis in Swine by Activating the LOX-1-Dependent Mitochondrial Pathway and Caspase-8-Dependent Pathway. J Cardiovasc Pharmacol Ther 2016;21:209-218.

-28 Park KM, Teoh JP, Wang Y, Broskova Z, Bayoumi A, Tang Y, Su H, Weintraub NL, Kim IM: Carvedilolresponsive microRNAs, miR-199a-3p and -214 protect cardiomyocytes from simulated ischemiareperfusion injury. Am J Physiol Heart Circ Physiol 2016;311:H371-H383.

29 Yan XC, Cao J, Liang L, Wang L, Gao F, Yang ZY, Duan JL, Chang TF, Deng SM, Liu Y: miR-342-5p Is a Notch Downstream Molecule and Regulates Multiple Angiogenic Pathways Including Notch, Vascular Endothelial Growth Factor and Transforming Growth Factor $\beta$ Signaling. J Am Heart Assoc 2016;5: e003042.

-30 Guedes EC, França GS, Lino CA, Koyama FC, Moreira LD, Alexandre JG, Barretochaves ML, Galante PA, Diniz GP: MicroRNA Expression Signature Is Altered in the Cardiac Remodeling Induced by High Fat Diets. J Cell Physiol 2016; 231:1771-1783.

-31 Deng L, Blanco FJ, Stevens H, Lu R, Caudrillier A, Mcbride M, Mcclure JD, Grant J, Thomas M, Frid M: MicroRNA-143 Activation Regulates Smooth Muscle and Endothelial Cell Crosstalk in Pulmonary Arterial Hypertension. Circ Res 2015;117:870-883.

-32 Liu H, Ning Y, Fei Z, Jie Q, Ma D, Liu X, Cai G, Sheng L: Analysis of plasma miR-208a and miR-370 expression levels for early diagnosis of coronary artery disease. Biomed Rep 2016;5:332-336.

33 Pilbrow AP, Cordeddu L, Cameron VA, Frampton CM, Troughton RW, Doughty RN, Whalley GA, Ellis CJ, Yandle TG, Richards AM: Circulating miR-323-3p and miR-652: candidate markers for the presence and progression of acute coronary syndromes. Int J Cardiol 2014;176:375-385

-34 Wang XH, Qian RZ, Zhang W, Chen SF, Jin HM, Hu RM: MicroRNA-320 expression in myocardial microvascular endothelial cells and its relationship with insulin-like growth factor- 1 in type 2 diabetic rats. Clin Exp Pharmacol Physiol 2010;6:181-188.

-35 Ghanbari M, Franco OH, de Looper HW, Hofman A, Erkeland SJ, Dehghan A: Genetic Variations in MicroRNA-Binding Sites Affect MicroRNA-Mediated Regulation of Several Genes Associated With Cardiometabolic Phenotypes. Circ Cardiovasc Genet 2015;8:473-486.

36 Wang K, Liu F, Zhou LY, Ding SL, Long B, Liu CY, Sun T, Fan YY, Sun L, Li PF: miR-874 regulates myocardial necrosis by targeting caspase-8. Cell Death Dis 2013;4:e709.

-37 Ning B, Zhang Y, Wu D, Cui J, Liu L, Wang P, Wang W, Zhu W, Chen Y, Zhang T: Luteolin-7-diglucuronide attenuates isoproterenol-induced myocardial injury and fibrosis in mice. Acta Pharmacol Sin 2017;38:331341.

-38 Wang Y, Ouyang M, Wang Q, Jian Z: MicroRNA-142-3p inhibits hypoxia/reoxygenation-induced apoptosis and fibrosis of cardiomyocytes by targeting high mobility group box 1. Int J Mol Med 2016;38:1377-1386. 